\title{
Teaching and learning of ethics in Ophthalmology: The perceptions of undergraduate medical students
}

\author{
Rajiv Gupta1, Saurabh Shrivastava ${ }^{2}$, Shahzaib Rehan ${ }^{3}$, Prabhu Venkatesan ${ }^{4}$, Soumendra Sahoo $^{5, *}$ \\ ${ }^{5}$ Professor \& HOD, ${ }^{\mathbf{1 2 , 4 , 5}}$ Dept. Ophthalmology. Melaka Manipal Medical College, Manipal Academy of Higher \\ Education(MAHE), Melaka, Malaysia, ${ }^{3}$ University Hospital of Wales, Cardiff, United Kingdom
}

*Corresponding Author:

Email: soumendra.sahoo@manipal.edu.my

\begin{abstract}
Purpose of Study: This study was aimed to uncover the perceptions of undergraduate students specifically pertaining to their experiences of ethics teaching and their opinions of the value of such teaching.

Methodology: We used a mixed methods design involving 148 students through a sequential exploratory strategy. Three ethics sessions revolved around the teaching of ethics in Ophthalmology after which the participants were interviewed using openended questions. The data was analysed by coding, categorisation and the subsequent derivation of themes. The quantitative analysis was done through a questionnaire survey to further explore the students' preference on teaching methodologies.

Results: Majority students perceive the objective of ethics teaching is to help them to become a better doctor, to better equip them to deal with ethical dilemmas in the future, to improve their social skills, and to help them to avoid legal complications. The preferred formats of learning ethics as expressed by participants was scenario based discussion (29\%), debate (25\%), selfdirected learning $(24 \%)$ \& lectures $(22 \%)$.

Conclusion: The common theme emerged from the qualitative analysis was that students wanted to learn ethics in order to help to make them better doctors. Scenario based discussion was the indicated preferred teaching method expressed by students.
\end{abstract}

Keyword: Ethics, Teaching, Students, Perception.

\section{Introduction}

Ethical practices in healthcare are driven by various oaths and declarations. ${ }^{1}$ Perhaps the most widely recognised oath amongst students and doctors in the medical profession, which has been recited by newly graduating physicians for centuries, is the Hippocratic Oath. ${ }^{2}$ Healthcare ethics has four commonly accepted principles: beneficence, nonmaleficence, autonomy and justice. Such principles i.e. doing good, avoiding harm, respecting patients and affording them autonomy, provide a framework for all practising doctors, and naturally advocate working in partnership with patients to guide care to try to achieve optimal outcomes. For the preceding reasons teaching and learning ethics in Ophthalmology, as per clinical medicine per se, is important, arguably fundamental and imperative.

Students in the medical profession should have a basic curriculum in ethics according to several groups and countries. ${ }^{3}$ However, due to the modernisation of curricula and time pressures, time devoted to ethics teaching has reduced at undergraduate level. The onus then lies with postgraduate training programmes to educate doctors and to deliver key messages. For example, doctors are privileged and have a profound responsibility and must therefore respect ethical principles. ${ }^{4}$ One study found that Ophthalmology residents in Canada rated ethics as being equally important to any other aspect of their curriculum. ${ }^{5}$

In the present study we wanted to find out our undergraduate students' perception of the value of learning ethics and their opinion of the value of such teaching.

\section{Methodology}

This study was mixed methods design using a sequential exploratory strategy. A total of 148 fourth year medical students participated in this study through total population sampling concept. The ethics sessions were revolved around different teaching learning techniques such as didactic lecture, scenario based large group learning, debate by students and self-directed learning. All the teaching learning topics revolved around ethical dilemmas related to ophthalmic practice. All of the study participants were interviewed using open-ended questions via a self-semi-structured interview format with an interviewed guide being provided for them. The qualitative data was analysed by coding, categorisation and the subsequent derivation of themes. The quantitative analysis was done using a questionnaire survey to further explore the students' preferences on teaching methodologies. The study has the approval of institutional research and ethics committee. 


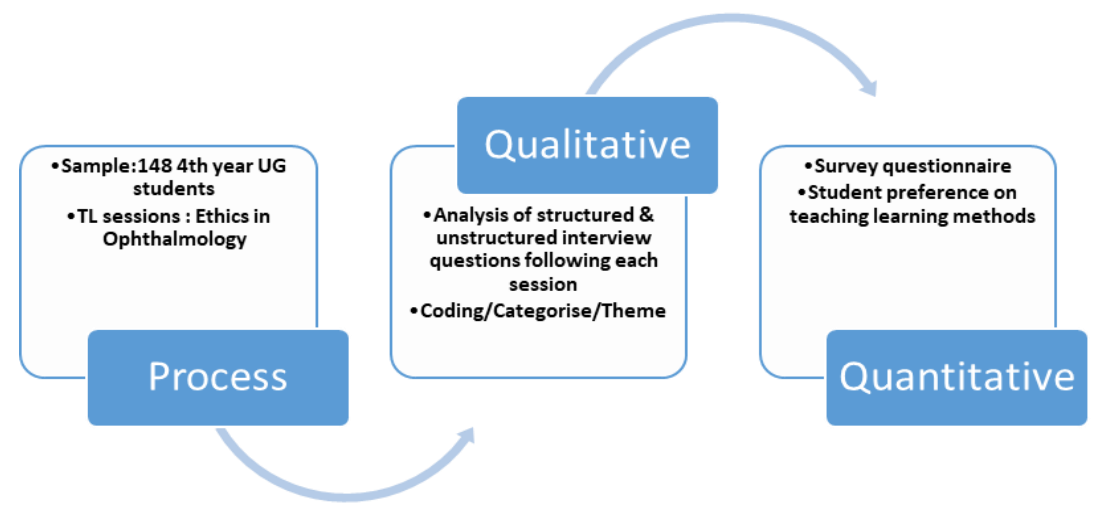

Fig. 1: Data collection method

Results

The demographics of participating students is depicted in Table 1.

Table 1: Demography of participants

\begin{tabular}{|l|c|}
\hline \multicolumn{1}{|c|}{ Variables } & $\begin{array}{c}\text { Participants }(\mathbf{n = 1 4 8}) \\
\text { n (\%) }\end{array}$ \\
\hline \multicolumn{2}{|c|}{ Age in years } \\
\hline$<20$ & $24(16.21 \%)$ \\
\hline$>20$ & $124(83.79 \%)$ \\
\hline \multicolumn{2}{|c|}{ Gender } \\
\hline Female & $98(66.21 \%)$ \\
\hline Male & $50(33.79 \%)$ \\
\hline \multicolumn{2}{|c|}{ Ethnicity } \\
\hline Malay & $46(31.78 \%)$ \\
\hline Chinese & $36(24.31 \%)$ \\
\hline Indian & $65(43.91 \%)$ \\
\hline Other & 1 \\
\hline
\end{tabular}

We wanted to know the perception of medical students with regard to medical ethics and how relevant and appropriate they considered ethics teaching in Ophthalmology by analysing the qualitative data. Some of the key words that emerged from the qualitative data on teaching ethics in Ophthalmology were: 'interesting', 'dealing with dilemmas', 'providing problem solving skills', 'communication skills', 'team work' and 'time constraints'. The major codes were further classified into two categories which were learning enhancement and dealing with legal issues. These categorizations subsequently led to the emergence of major themes such as to become a good doctor and to protect oneself from one being sued. Fig. 2.

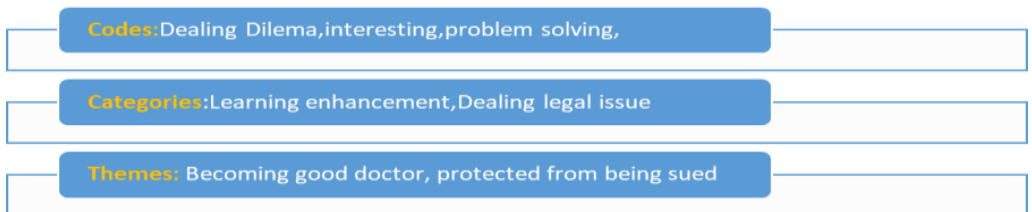

Fig. 2: Flow of Qualitative Analysis

In response to the question 'why are you interested in learning ethics?' some of the responses given were: to help them to become a better doctor, to better equip them to deal with ethical dilemmas in the future, to improve their social skills, and to help them to avoid legal complications. One student stated that the key driver for understanding ethical and legal principles was in order to prevent them from being 'sued'. Some students expressed a desire to receive no formal ethics teaching. However, they indicated that acquiring practical problem solving skills on ethical dilemmas was desirable. Around 7\% of students were not interested in learning about ethics during their undergraduate training. A small number considered ethics to be a complicated subject matter and a few believed that ethics was an innate value which required no active learning. Further exploration of student's perception through a 
questionnaire survey is summarised in Table 2. This indicates that the learners prefer to have ethics teaching sessions and multiple formats are liked by majority of participants.

Table 2: Analysis of Students' perception of Ethic Sessions in Ophthalmology

\begin{tabular}{|l|c|c|c|c|c|}
\hline \multicolumn{1}{|c|}{ Statements } & \multicolumn{5}{|c|}{ No \& \% of students responded: Strongly disagree(1), } \\
Strongly agree(5)
\end{tabular}

The preferred format for the teaching and learning of ethics as expressed by participants was: $29 \%$ for scenario based discussions, $25 \%$ for debates, $24 \%$ for self-directed learning, and $22 \%$ for a lecture based programme/approach as depicted in Fig. 3.

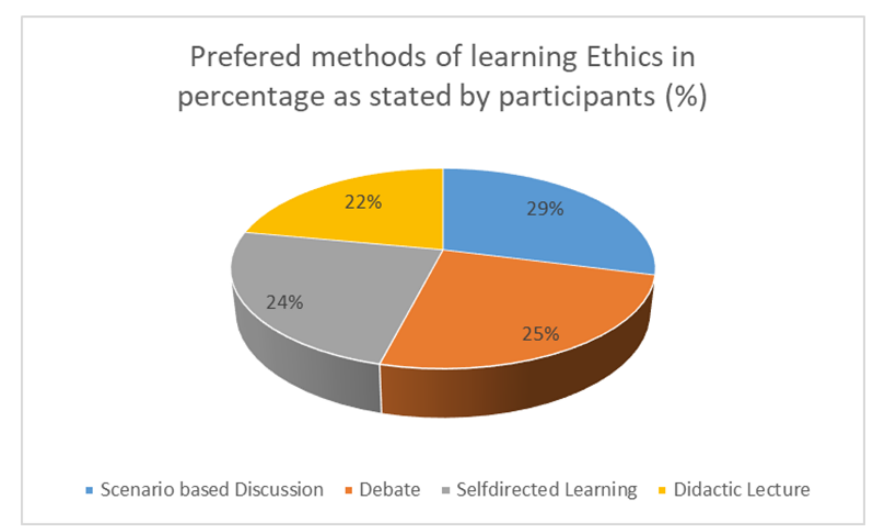

Fig. 3: Students' preference on the methods of teaching Ethics

\section{Discussion}

Learning ethics is important and arguably helps to make individuals better doctors. This emerged as a common theme from our qualitative analysis. It also equips doctors with the necessary skills to deal with the dilemmas faced in day to day clinical practice. ${ }^{6}$ However, some authors, such as Smith (2002) are of the view that teaching ethics to Ophthalmology residents in a structured curriculum is of no value. ${ }^{7}$ In his work he has cited Hundert EM (1996) who said, "Ethics can't be taught....but it can be learned". Janson, CG (1996) suggested that ethics should not be "taught" in medical school (or residency) and should only be encouraged by discussion and through self-reflections. Moreover, role models to emulate have a role. Sapeth GL (2003) while commenting on the American Academy of Ophthalmology's primer on Ethical behaviour noted that the aim of the primer is to help foster ethical 
behaviour. But the learning of the substance of the text by itself is of little or no help in that regard. He summarised his views as follows: 1) the subject of ethics is taught by those knowledgeable in the subject of ethics and is learned by those who have the capability and intention to learn and are exposed to appropriate teachers, 2. ethical behaviour is taught by those who behave ethically and is learned by those who have the capability and the intention to learn and are exposed to individuals who behave ethically. ${ }^{8}$

Scenario based discussions were expressed as being the preferred format for the teaching and learning of ethics. Samuel Packer (2005) used the Defining Issues Test (DIT-2) developed by James Rest which involves answering standardized questions about four moral dilemmas. The results of the DIT- 2 taken before and after the ethics lectures were compared. ${ }^{9} \mathrm{He}$ concluded that the effect of an ethics course on the resident's ability to answer moral dilemmas did not achieve statistical significance. This matches with our study with regards to the students' preference on the mode of teaching and thus learning.

In a study by Carolyn Johnston ${ }^{10}$ et al undergraduate students when questioned mentioned the following views with respect to the merits of teaching medical ethics: to help them to become better doctors $(\mathrm{n}=219,92 \%)$, to prevent them from being sued $(\mathrm{n}=169$, $71 \%)$ and to help them to develop problem-solving skills $(\mathrm{n}=123,52 \%)$. Most of their emerging themes match with the themes found from our qualitative analysis. In the same study with regards to the format of teaching ethics, the majority of students indicated that they prefer lectures and break-out sessions (77 out of $129(60 \%))$. The other results were as follows: 48 out of 129 (37\%) prefer small-group sessions only, whereas 4 out of $129(3 \%)$ prefer individual problem-based learning with virtual campus support. In contrast our study reflected the students' preference towards scenario based learning to didactic lectures.

In another study conducted in the United Arab Emirates $^{11}$ (UAE), the responses of students showed a positive correlation between the extent of ethics education and its usefulness in dealing with ethical conflicts $(r=0.642, \mathrm{P}<0.000)$. There was a correlation between encountering ethical conflicts during training and the attitudes towards the subject of ethics (aggregates score $\mathrm{r}=0.225, \mathrm{P}<0.05$ ). That matches with one of the qualitative responses from our participants.

The strength of our study is the mixed methods design that we deployed. However the limitation is that we did not correlate various modes of teaching to academic performance reflecting professionalism. That will be taken up in the follow up study.

\section{Conclusion}

The common theme that emerged from the qualitative analysis was that students wanted to learn ethics in order to help to make them better doctors. Scenario based discussion was the indicated preferred teaching method expressed by students.

\section{References}

1. Gabel S. Ethics and values in clinical practice: Whom do they help? Mayo Clin Proc 2011;86(5):421-24.

2. Sritharan K, Russell G, Fritz Z, Wong D, Rollin M, Dunning J, et al. Medical oaths and declarations: a declaration marks an explicit commitment to ethical behaviour. BMJ 2001;323(7327):1440-41.

3. Lakhan, S.E., Hamlat, E., McNamee, T. et al. Philos Ethics Humanit Med 2009;4:13. https://doi.org/10.1186/1747-5341-4-13

4. Day SH. A structured curriculum on ethics for Ophthalmology residents is valuable. Arch Ophthalmol 2002;120(7):963-64.

5. Hope WC, LaRoche R, Levin AV. Teaching ethics to Ophthalmology residents: the Halifax Symposium. Can J Ophthalmol 2016;51(3):188-89.

6. Clarke MP. There are Ethical issues in Ophthalmology. BMJ 1999;318:1153

7. Smith ME. A Structured Curriculum on ethics for Ophthalmology residents is not valuable. Arch Ophthalmol 2002;20:965

8. Sapeth GL. Teaching and learning ethics. Arch Ophthalmol 2003;121:1342

9. Packer S. The Ethics education of Ophthalmology residents: An experiment. Trans Am Ophthalmol Soc 2005;103:240-69.

10. Carolyn Johnston, Peter Haughton. Medical students' perceptions of their ethics teaching. $J$ Med Ethics 2007;33:418-22.

11. Tahra AlMahmoud, M. Jawad Hashim, Margaret Ann Elzubeir \& Frank Branicki. Ethics teaching in a medical education environment: preferences for diversity of learning and assessment methods. Med Educ Online 2017;22(1): 1328257. doi: 10.1080/10872981.2017.1328257 